\title{
EFICACIA IN VITRO E IN SITU DE PRODUCTOS ANTIMANCHA SIN FENOLES CLORADOS.
}

\author{
David Saelzer C. ${ }^{(*)}$ \\ Miguel Peredo L. $(* *)$
}

\section{RESUMEN}

El estudio de productos que puedan reemplazar al PCP-Na en la prevención de la rancha azul del pino requiere de métodos de laboratorio que permitan encontrar la osis minime efectiva (d.m.e.) de un preservante, in vitro, y asi obtener el rango de incentraciones que debe ser empleado en ensayos in situ. De modo que, es uportante saber si la d.m.e. obtenida en una prueba de laboratorio se acerca a los ysultados de terreno. En este trabajo se prueba la eficacia de dos preservantes intimancha alternativos al PCP-Na en la prevención del ataque de Ceratocystis pilifera fries) C. Moreau, sobre madera de Pino Pinus radiata D. Don), según las sspecificaciones de la norma ASTM D 4445-84. Se compara los resultados, con los que ia obtiene de un ensayo montado en la cancha de secado de un aserradero. BUSAN '009 y SINESTO B son efectivos en laboratorio al $2.0 \%$ y $5.0 \%$, respectivamente. En irreno, la d.m.e. de ambos preservantes no supera estos valores.

2alabras claves : Pinus radiata, Presenvación y Baño antimancha

"Ingeniero Forestal. Casilla 68-D, Valdivia.

") Ingeniero Forestal. Dr. Ciencias Forestales. Instituto de Tecnologia de Productos Forestales.

U.A.CH. Casilla 853, Valdivia. 


\section{ABSTRACT}

The study of products that can replace PCP-Na in the prevention of blue stan requieres the use of laboratory methods to determine the effective minimal dosis (e.m.d) of a preservative, in vitro, so as to calculate the concentration range that can be used on trials in situ. It is important therefore to know whether the e.m.d. obtained in laboratory are similar to the results obtained in the field. This research proves the effectiveness $d^{\prime}$ two alternative products, which do not present clored fenols, in preventing the attact of Ceratocystis pilifera (Fries) C. Moreau, over pine wood (Pinus radiata D. Don). according with specifications of norm ASTM D 4445-84. Result are compared with those obtained in a trial established in a storeyard of a sawmill. BUSAN 1009 and SINESTO are effective in laboratory at $2.0 \%$ and $5.0 \%$ respectively. In the field, the e.m.d. of both preservatives do not surpass these values.

Keywords: Pinus radiata, Preservation and anti sapstrain dip. 


\section{INTRODUCCION}

Debido a la importancia de la madera aserrada de pino insigne (Pinus radiata $\mathrm{D}$. Don) en la industria nacional del aserrío y la prohibición del uso de pentaclorofenato de sodio ( $\mathrm{PCN}-\mathrm{Na}$ ) en muchos paises que importan esta madera (David, 1991; C.E.E., 1991), se han realizado numerosos estudios destinados a evaluar la eficacia de productos antimancha que puedan sustituir al PCP-Na en la prevención del azulado del pino (Hanke, 1991; Quiroz, 1991; Peña, 1988). En este tipo de estudios se requiere de métodos fidedignos, para probar los diferentes preservantes, que sean representativos de las condiciones naturales en que los hongos manchadores se desarrollan y que a la vez puedan implementarse en laboratorio en forma más o menos simple, sin tardar demasiado en obtener los resultados (Schulz, 1951).

Luego de obtener la dosis mínima efectiva (d.m.e.) de un producto en laboratorio, se debe repetir el ensayo de eficacia en terreno, ya que las condiciones reales en que la madera debe ser protegida de la mancha azulalmacenaje en aserradero, puerto de embarque y transporte maritimo - son sensiblemente diferentes a las condiciones de laboratorio (Williams, 1991a). La obtención de resultados en un ensayo preliminár de terreno tarda cerca de 90 dias de manera que, si las concentraciones ensayadas en terreno - a partir de los datos obtenidos en laboratorio - no corresponden al rango en que se encuentra la d.m.e., se puede perder varios meses de experimentación.

El objetivo de este trabajo es determinar la d.m.e. in vitro de dos preservantes antimancha alternativos al PCP-Na, y comparar los resultados, con los obtenidos mediante una prueba de eficacia in situ. 


\section{MATERIAL Y METODO}

\section{Material}

Se utilizó madera fresca de albura de pino insigne (Pinus radiata D. Don)., sin manchas ni defectos que puedan alterar los resultados. Para el ensayo de laboratorio se dimensionaron probetas de corte tangencial, de $70 \times 20 \times 7 \mathrm{~mm}$ (ASTM, 1984). En el ensayo montado en terreno se ocupó probetas de $2,5 \mathrm{~m}$ de largo $\times 100$ a $150 \mathrm{~mm}$ de ancho $\times 25,4 \mathrm{~mm}$ de espesor.

En la prueba de eficacia de laboratorio se utilizó Ceratocystis pilifera (Fries) C. Moreau como agente manchador. En el ensayo de terreno la madera se dejó en un lugar que aseguraba su contaminación con hongos manchadores. En las dos pruebas se trató la madera con los preservantes BUSAN 1009 y SINESTO B. Las concentraciones aplicadas se presentan en el Cuadro $\mathrm{N}^{\circ} 1$.

\section{Cuadro $\mathrm{N}^{\circ} 1$}

CONCENTRACIONES DE BUSAN 1009 Y SINESTO B APLICADAS EN LOS TRES ENSAYOS (\% p/p)

\begin{tabular}{|l|l|c|}
\hline Producto & Ensayo & Concentración \\
\hline \multirow{3}{*}{$\begin{array}{l}\text { Busan } \\
1009\end{array}$} & Laboratorio & $0,5 \quad 1,01,5 \quad 2,0 \quad 2,5 \quad-$ \\
\cline { 2 - 4 } & Terreno & $-1,5 \quad 2,0-3,0 \quad 4,0$ \\
\hline \multirow{2}{*}{$\begin{array}{l}\text { Sinesto } \\
\text { B }\end{array}$} & Laboratorio & $4,04,5 \quad 5,05,5 \quad 6,0$ \\
\cline { 2 - 3 } & Terreno & $-4,55,0-6,0 \quad 8,0$ \\
\hline
\end{tabular}

(-) concentraciones no ensayadas

En la prueba de eficacia in vitro se utilizó además PCP-Na al $2 \%$ como producto patrón. 


\section{Método}

El ensayo de laboratorio se realizó siguiendo las especificaciones de la norma ASTM D 4445-84 (ASTM, 1984). Para cada concentración de los preservantes analizados, se utilizó 10 repeticiones, 10 mismo para el producto patrón y para el testigo, lo que hace un total de 120 probetas.

Las probetas se trataron con el producto a la concentración correspondiente $(p / p)$, mediante inmersión por 15 segundos en la solución preservante contenida en un vaso de precipitado. Las probetas testigo fueron bañadas en agua destilada para mantener el mismo grado de humedad en la madera.

Después del baño se dejó estilar las probetas por 10 segundos, y luego se introdujo de a dos probetas en cada placa petri, provista de tres láminas de papel filtro bajo una plca de aluminio ( $3 \mathrm{~mm}$ espesor). Todo el material preparado fue esterilizado a $121^{\circ} \mathrm{C}$ y $1 \mathrm{Kg} / \mathrm{cm}^{2}$ de presión durante 15 minutos para lograr el crecimiento exclusivo del hongo de prueba.

Después de $\mathbf{2 4}$ horas, las probetas fueron inoculadas en la cara superior con $2 \mathrm{ml}$ de una solución de micelio y esporas del hongo de prueba. Se agregó agua destilada hasta cubrir los papeles filtro con una delgada película de agua, y asi mantener una humedad ambiental adecuada para el desarrollo del hongo. Con este mismo propósito, se envolvió cada 5 o 6 placas en una bolsa de polietileno.

Por último, las probetas se dejaron por cuatro semanas en una estufa climatizada a $25^{\circ} \mathrm{C}$ aproximadamente, donde se les continuó aplicando agua esterilizada de acuerdo a la necesidad de cada placa petri. Se realizaron cuatro mediciones - cada una semana - de las superficies con crecimiento de micelio sobre las probetas. En la última medición, se registró además la superficie con presencia de mancha azul. Las mediciones se hicieron con la ayuda de una malla cuadriculada que dividia la superficie de las probetas en 20 partes iguales.

En los ensayos de terreno se utilizaron 30 probetas por cada concentración, más 60 probetas tetigo, lo que suma 300 repeticiones. Las probetas de madera fresca recién aserrada fueron bañadas durante 15 segundos en las soluciones preservantes antimancha y luego se dejaron escurrir sobre el recipiente que contenia la solución. La preparación de cada concentración se hizo al finalizar la aplicación de la concentración inmediatamente superior, añadiendo el agua 
necesaria para diluirla. En el cálculo consideró la absorción de 64 lts. de solución por cada 100 pulgadas de madera tratada.

La madera fue almacenada durente 90 dias, entre el 17.12 .92 y el 17.02.93, mediante dos sistemas de encastillado: con y sin separador, en el Patio del Aserradero Vista Alegre Ltda. en Valdivia. La evaluación de la superficie manchada se hizo mediante inspección ocular de la cara y trascara de las probetas. La superficie e intensidad de mancha se clasificó en rangos de $0 \%$, $1-10 \%, 11-25 \%, 26-50 \%$ y $>50 \%$. Se realizaron tres mediciones separadas por 30 días.

En la Figura $\mathrm{N}^{\circ} 1$, se muestra el diseño experimental utilizado en el ensayo de terreno. La parte superior (A), representa un bloque, donde cada una de las 30 probetas está identificada con una letra y un número. Las líneas horizontales indican la presencia de separadores entre las probetas. En la segunda parte de la figura (B) se explica la disposición de la madera encastillada. Los dos primeros castillos constan de cuatro bloques, como el anteriormente descrito. Cada uno de estos bloques está compuesto de 30 probetas tratadas con un producto a una concentración determinada. El tercer castillo tiene sólo dos bloques, ambos con madera sin tratar.

A)

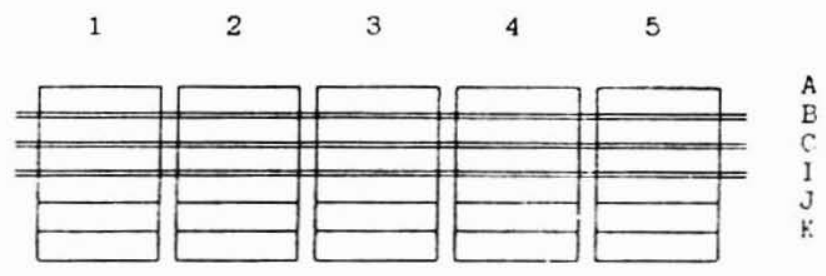

\begin{tabular}{|c|c|c|}
\hline BUSAN 2.0\% & SINESTO $5.0 \%$ & \\
\hline BUSAN 3.07 & SINESTO $5.5 \%$ & TES SUP \\
\hline BUSAN $4.0 \%$ & SINESTO $8.0 x$ & TES INF \\
\hline
\end{tabular}

Figura No 1 . DISEÑO DE LOS CASTILLOS DEL ENSAYO EN TERRENO (TES, SUP Y TES INF: TESTIGO, BLOQUE SUPERIOR E INFERIOR) 
A partir de los resultados obtenidos en los ensayos realizados, se comparó la efectividad de los preservantes antimancha según la d.m.e. Para ésto, se aceptó como máximo un promedio de $5 \%$ de la superficie total manchada. Bajo este límite, una concentración cualquiera se consideró efectiva en el tratamiento contra el azulado del pino.

Además se comparó la tendencia en el comportamiento de la curva : superficie promedio manchada $\mathrm{v} / \mathrm{s}$ concentración aplicada, con el fin de comprobar si la efectividad, como variable dependiente de la concentración, se comporta de la misma forma en terreno que en laboratorio.

\section{RESULTADOS}

En el Cuadro $\mathrm{N}^{\circ} 2$ se presentan en forma resumida los resultados obtenidos al finalizar los ensayos montados en laboratorio y en terreno. En este último los resultados aparecen divididos según el tipo de encastillado : con separador y $\sin$ separador.

\section{Ensayo de Laboratorio}

Las probetas tratadas con BUSAN 1009 al $05 \%$ presentaron desarrollo de micelio desde la primera semana de ensayo. La superficie promedio afectada en cada medición fue : $2 \%, 18 \%, 23 \%$ y $26 \%$. Las probetas tratadas al $1 \%$ y $1,5 \%$ sólo representaron mancha, mientras que las concentraciones $2 \%$ y 2,5 fueron efectivas en un $100 \%$ (Cuadro $N^{\circ} 3$ ). 


\section{Cuadro $\mathrm{N}^{\circ} 2$}

SUPERFICIE PROMEDIO DE MADERA MANCHADA, TRATADA CON BUSAN 1009 Y SINESTO B A DISTINTAS CONCENTRACIONES (\%)

\begin{tabular}{|c|c|c|c|}
\hline \multirow{3}{*}{ Tratamiento } & \multicolumn{3}{|c|}{ Método } \\
\hline & \multirow[t]{2}{*}{ Laboratorio } & \multicolumn{2}{|c|}{ Terreno } \\
\hline & & Con separador & Sin separador \\
\hline $\begin{array}{l}\text { BUSAN } 0,5 \% \\
\text { BUSAN } 1,0 \% \\
\text { BUSAN } 1,5 \% \\
\text { BUSAN } 2,0 \% \\
\text { BUSAN } 2,5 \% \\
\text { BUSAN } 3,0 \% \\
\text { BUSAN } 4,0 \%\end{array}$ & $\begin{array}{r}58,0 \\
20,0 \\
18,5 \\
0.0 \\
0.0 \\
. \\
-\end{array}$ & $\begin{array}{r}: \\
3.0 \\
0,0 \\
0.0 \\
0,0\end{array}$ & $\begin{array}{r}\dot{.} \\
17,9 \\
4,7 \\
0.5 \\
0,9\end{array}$ \\
\hline $\begin{array}{l}\text { SINESTO } 4,0 \% \\
\text { SINESTO } 4,5 \% \\
\text { SINESTO } 5,0 \% \\
\text { SINESTO } 5,5 \% \\
\text { SINESTO } 6,0 \% \\
\text { SINESTO } 8,0 \%\end{array}$ & $\begin{array}{r}20,1 \\
22,5 \\
0,0 \\
0,0 \\
0,0 \\
\end{array}$ & $\begin{array}{r}11,9 \\
1,5 \\
0 \\
0,7 \\
0,0\end{array}$ & $\begin{array}{l}4,6 \\
3,1 \\
1,7 \\
0,0\end{array}$ \\
\hline $\begin{array}{l}\text { PCF-Na } \\
\text { Testigo }\end{array}$ & $\begin{array}{r}0,0 \\
100,0\end{array}$ & 28,9 & $74, \overline{1}$ \\
\hline
\end{tabular}

(-) Concentraciones no ensayadas

\section{Cuadro $\mathrm{N}^{\circ} 3$}

SUPERFICIE ATACADA EN LA CUARTA MEDICION EN PROBETAS TRATADAS CON BUSAN 1009 (\%)

\begin{tabular}{|c|rrrrrrrrrr|r|r|}
\hline CC & \multicolumn{10}{|c|}{ Repeticiones } & MED \\
\cline { 2 - 11 } & 1 & 2 & 3 & 4 & 5 & 6 & 7 & 8 & 9 & 10 & \\
\hline $0,5 \%$ & 95 & 0 & 85 & 0 & 0 & 100 & 100 & 0 & 100 & 100 & 58,0 \\
$1,0 \%$ & 0 & 0 & 100 & 0 & 0 & 100 & 0 & 0 & 0 & 0 & 20,0 \\
$1,5 \%$ & 35 & 50 & 0 & 0 & 0 & 100 & 0 & 0 & 0 & 0 & 18,5 \\
$2,0 \%$ & 0 & 0 & 0 & 0 & 0 & 0 & 0 & 0 & 0 & 0 & 0,0 \\
$2,5 \%$ & 0 & 0 & 0 & 0 & 0 & 0 & 0 & 0 & 0 & 0 & 0,0 \\
\hline
\end{tabular}


Ninguna de las concentraciones aplicadas de SINESTO B permitió al hongo desarrollar micelio sobre probetas, sin embargo, varias repeticiones tratadas con el preservante al $4 \%$ y $4,5 \%$ resultaron manchadas (Cuadro $\mathrm{N}^{\circ} 4$ ).

Las probetas tratadas con PCP-Na al 2,0\% no registraron crecimiento de micelio ni presencia de mancha azul. En cambio, todas las probetas testigo presentaron crecimiento de micelio en un $100 \%$ desde la segunda medición.

\section{Cuadro $\mathrm{N}^{\circ} 4$}

\section{SUPERFICIE ATACADA EN LA CUARTA MEDICION EN PROBETAS} TRATADAS CON BUSAN 1009(\%)

\begin{tabular}{|c|c|c|c|c|c|c|c|c|c|c|c|}
\hline \multirow[t]{2}{*}{$\mathrm{CC}$} & \multicolumn{10}{|c|}{ Repeticiones } & \multirow[t]{2}{*}{ MED } \\
\hline & 1 & 2 & 3 & 4 & 5 & 6 & 7 & 8 & 9 & 10 & \\
\hline $4,0 \%$ & 0 & 0 & 1 & 0 & 0 & 0 & 50 & 100 & 100 & 100 & 20,1 \\
\hline $4,5 \%$ & 0 & 0 & 0 & 40 & 10 & 0 & 0 & 0 & 80 & 95 & 22,5 \\
\hline $5 \%-6 \%$ & 0 & 0 & 0 & 0 & 0 & 0 & 0 & 0 & 0 & 0 & 0,0 \\
\hline
\end{tabular}

\section{Ensayo en terreno}

El producto BUSAN 1009 fue efectivo en todas las concentraciones aplicadas, excepto en la madera tratada al $1,5 \%$, encastillada sin separador, cuya superficie se manchó en más de un $5 \%$, desde la primera medición. En los demás casos, la madera no se manchó o se manchó en un porcentaje promedio muy cercano a cero, y las probetas presentaron casi en su totalidad una superficie manchada menor al $10 \%$ (Cuadro $N^{\circ} 5$ ). 


\section{Cuadro $N^{\circ} 5$}

FRECUENCIA Y PROMEDIO DE SUPERFICIE MANCHADA EN MADERA TRATADA A DISTINTAS CONCENTRACIONES DE BUSAN 1009.(\%)

\begin{tabular}{|c|c|c|c|c|c|c|c|c|}
\hline CC\% & \multicolumn{2}{|c|}{ Superficie } & $0 \%$ & $1.10 \%$ & $11-25 \%$ & $26-50 \%$ & $>50 \%$ & Media \\
\hline \multirow{3}{*}{1.5} & $\mathrm{C} / \mathrm{S}$ & Cara & 60 & 40 & 0 & 0 & 0 & 2.0 \\
\hline & & Tras & 73 & 27 & 0 & 0 & 0 & 1.3 \\
\hline & \multicolumn{2}{|c|}{$\mathrm{S} / \mathrm{S}$} & 30 & 20 & 20 & 25 & 5 & 17.9 \\
\hline \multirow{3}{*}{2,0} & $\mathrm{C} / \mathrm{S}$ & Cara & 100 & 0 & 0 & 0 & 0 & 0.0 \\
\hline & & Tras & 100 & 0 & 0 & 0 & 0 & 0.0 \\
\hline & \multicolumn{2}{|c|}{$\overline{S / S}$} & 20 & 75 & 5 & 0 & 0 & 4,7 \\
\hline \multirow{3}{*}{3.0} & $\mathrm{C} / \mathrm{S}$ & Cara & 100 & 0 & 0 & 0 & 0 & 0,0 \\
\hline & & Tras & 100 & 0 & 0 & 0 & 0 & 0.0 \\
\hline & \multicolumn{2}{|c|}{$\mathrm{S} / \mathrm{S}$} & 90 & 10 & 0 & 0 & 0 & 0,5 \\
\hline \multirow{3}{*}{4,0} & $\mathrm{C} / \mathrm{S}$ & Cara & 100 & 0 & 0 & 0 & 0 & 0,0 \\
\hline & & Tras & 100 & 0 & 0 & 0 & 0 & 0.0 \\
\hline & \multicolumn{2}{|c|}{$\frac{1}{S / S}$} & 95 & 0 & 5 & 0 & 0 & 0,9 \\
\hline
\end{tabular}

C/S : con separadores

S/S: sin separadores

CC\% : concentración

El cálculo del promedio de superficie atacada, en los Cuadros $N^{\circ} 5$ y $N^{\circ} 6$, se hizo en base a la ponderación de cada marca de clase por la frecuencia de probetas respectiva.

En la madera tratada con SINESTO B al 4,5\% encastillada con separadores, se manchó sobre el $5 \%$ de la superficie desde la primera semana. En todos los demás casos el producto fue efectivo en la prevención de la mancha azul, durante los tres meses de ensayo (Cuadro $\mathrm{N}^{\circ} 6$ ). 


\section{Cuadro $\mathrm{N}^{\circ} 6$}

FRECUENCIA Y PROMEDIO DE SUPERFICIE MANCHADA EN MADERA

TRATADA A DISTINTAS CONCENTRACIONES DE SINESTO B (\%)

\begin{tabular}{|c|c|c|c|c|c|c|c|c|}
\hline CC\% & \multicolumn{2}{|c|}{ Superficie } & $0 \%$ & $1-10 \%$ & $11-25 \%$ & $26-50 \%$ & $>50 \%$ & Media \\
\hline \multirow{3}{*}{4,5} & $\mathrm{C} / \mathrm{S}$ & Cara & 27 & 27 & 13 & 20 & 13 & 21,3 \\
\hline & & Tras & 87 & 13 & 0 & 0 & 0 & 0,7 \\
\hline & \multicolumn{2}{|c|}{$\mathrm{s} / \mathrm{S}$} & 75 & 15 & 0 & 10 & 5 & 4,6 \\
\hline \multirow{3}{*}{5.0} & $\mathrm{C} / \mathrm{S}$ & Cara & 86 & 7 & 7 & 0 & 0 & 1,5 \\
\hline & & Tras & 93 & 7 & 0 & 0 & 0 & 0,3 \\
\hline & \multicolumn{2}{|c|}{ S/S } & 65 & 25 & 10 & 0 & 0 & 3,1 \\
\hline \multirow{3}{*}{6.0} & $\mathrm{C} / \mathrm{S}$ & Cara & 93 & 7 & 0 & 0 & 0 & 0,3 \\
\hline & & Tras & 93 & 7 & 0 & 0 & 0 & 0,3 \\
\hline & \multicolumn{2}{|c|}{ S/S } & $80^{\circ}$ & 15 & 5 & 0 & 0 & 1,7 \\
\hline \multirow{3}{*}{8,0} & $\mathrm{C} / \mathrm{S}$ & Cara & 100 & 0 & 0 & 0 & $\overline{0}$ & 0.0 \\
\hline & & Tras & 100 & 0 & 0 & 0 & 0 & 0.0 \\
\hline & \multicolumn{2}{|c|}{ S/S } & 100 & 0 & 0 & 0 & 0 & 0,0 \\
\hline
\end{tabular}

C/S : con separadores

S/S: sin separadores

CC\% : concentración

La totalidad de las probetas testigo presentaron desarrollo de mancha azul, la mayoria desde la primera medición (Cuadro $N^{\circ} 7$ ). En la madera encastillada sin separador, asociada al azulado se registró abundante crecimiento de micelio, que cubría cerca de $100 \%$ de la superficie de las probetas, con un aspecto muy similar al que se desarrolló sobre las probetas en los ensayos de laboratorio. 


\section{Cuadro $\mathrm{N}^{\circ} 7$}

FRECUENCIA Y PROMEDIO DE SUPERFICIE MANCHADA EN MADERA SIN TRATAMIENTO (\%)

\begin{tabular}{|l|l|rrrrr|l|}
\hline \multicolumn{2}{|l}{ Superficie } & $0 \%$ & $1-10 \%$ & $\begin{array}{r}11- \\
25 \%\end{array}$ & $\begin{array}{r}26- \\
50 \%\end{array}$ & $>50 \%$ & Media \\
\cline { 1 - 5 } C/S & Cara & 0 & 7 & 30 & 30 & 33 & 42,1 \\
& Tras & 0 & 60 & 33 & 3 & 3 & 12,8 \\
\hline \multicolumn{2}{|c|}{ S/S } & 0 & 0 & 0 & 3 & 97 & 74,1 \\
\hline
\end{tabular}

$\mathrm{C} / \mathrm{S}$ : con separadores

S/S : sin separadores

CC\% : concentración

\section{Dosis minima efectiva}

Como se muestra en el Cuadro $N^{\circ} 8$, en ninguno de los casos la d.m.e. de BUSAN 1009 y SINESTO B resultó ser superior en terreno que en laboratorio.

\section{Cuadro $N^{\circ} 8$}

DOSIS MINIMA EFECTIVA in vitro E in situ DE LOS PRODUCTOS BUSAN 1009 U SINESTO B

\begin{tabular}{|c|c|c|c|}
\hline Producto & In vitro & \multicolumn{2}{|c|}{ In situ } \\
& & con sep. & sin sep. \\
\hline BUSAN 1009 & $2.0 \%$ & $1,5 \%\left(\left(^{\circ}\right)\right.$ & $2.0 \%$ \\
SINESTO B & $5.0 \%$ & $5.0 \%$ & $\left.4.5 \%()^{\circ}\right)$ \\
\hline
\end{tabular}

(") d.m.e. puede ser menor que esa cifra

Para BUSAN 1009 encastillado sin separador y SINESTO B con separador la d.m.e., usando como límite un $5 \%$ de superficie promedio manchada, fue la misma registrada en laboratorio según la norma ASTM D 445-84. En los otros dos casos sólo se puede inferir que la d.m.e. de estos productos aplicados en 
terreno, fue igual o menor que la d.m.e. obtenida in vitro, ya que no fueron ensayadas concentraciones menores.

\section{Curva efectividad v/s concentración}

Las Figuras $\mathrm{N}^{\circ} 2$ y $\mathrm{N}^{\circ} 3$ muestran la tendencia en el comportamiento de la superficie manchada influida por las concentraciones a que se aplica cada producto. Como se puede ver, la curva que correlaciona la concentración con la superficie manchada, en la prueba in vitro, cruza las demás curvas, que corresponden a la tendencia en el comportaminto del azulado observado en el ensayo in situ.

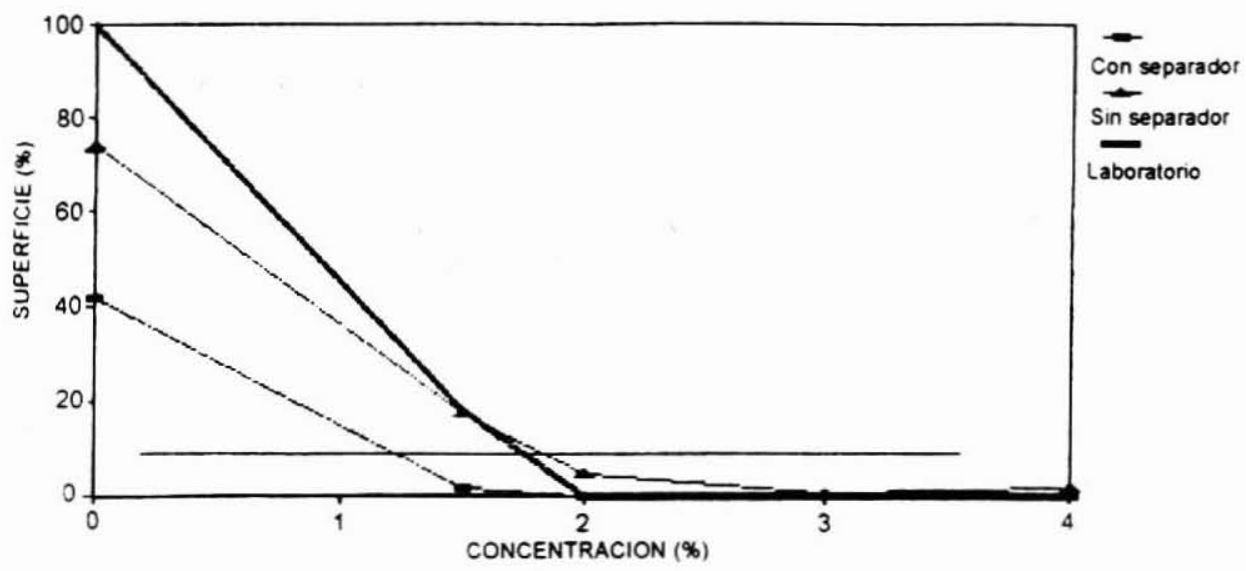

Figura $N^{\circ}$ 2. TENDENCIA EN EL COMPORTAMIENTO DE LA SUPERFICIE MANCHADA SEGUN LA CONCENTRACION DE BUSAN 1009

Se puede comprobar entonces, que la influencia de la concentración aplicada sobre los hongos causantes del azulado de la madera, es mayor en las condiciones que proporciona la prueba de eficacia en laboratorio. 
De acuerdo a esto, para predecir la eficacia en la prevención de mancha azul en terreno, la prueba in vitro sub-estima el efecto del producto en un rango de concentraciones bajas y lo sobreestima en un rango superior a la dosis minima efectiva.

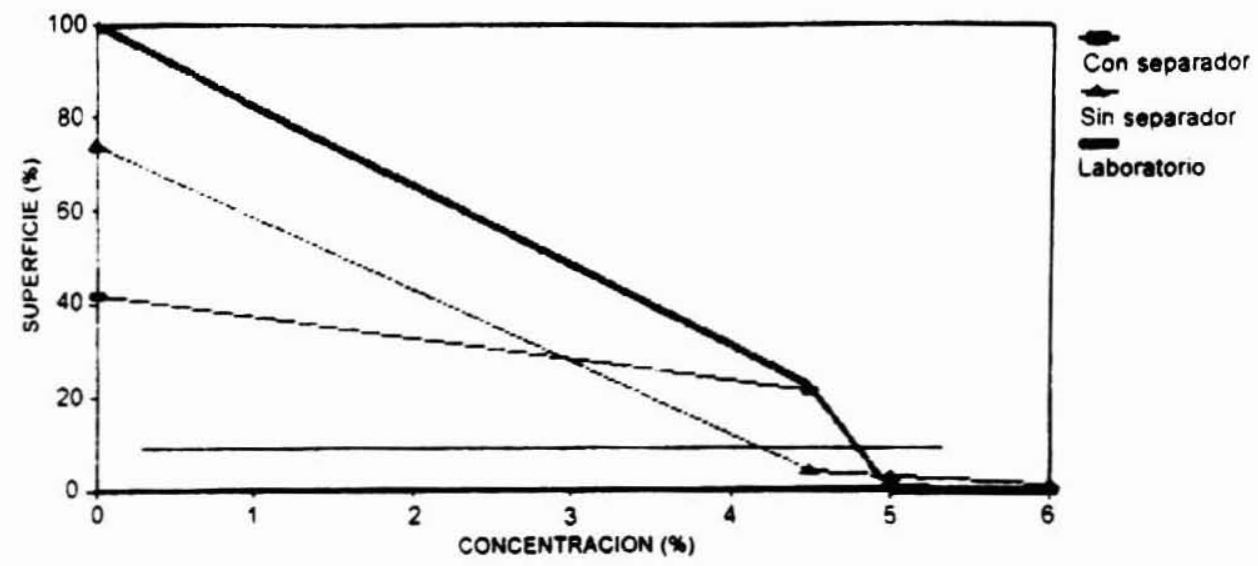

Figura $N^{\circ}$ 3. TENDENCIA EN EL COMPORTAMIENTO DE LA SUPERFICIE MANCHADA SEGUN LA CONCENTRACION DE SINESTO B

Debido a este comportamiento en las curvas que relacionan la superficie manchada y la concentración aplicada, la comparación de la d.m.e. observada en terreno y en laboratorio, sólo se puede hacer en base a la superficie promedio manchada que se acepte como límite: en este cso, $5 \%$ de la superficie total, como indica la linea horizontal en las Figuras $\mathrm{N}^{\circ} 2$ y $\mathrm{N}^{\circ} 3$. 


\section{DISCUSION}

\section{Sustrato y ambiente de cultivo}

La estufa empleada, cuya temperatura oscilaba entre los 28,5 y 21 , y las bolsas de polietileno no se ajustan integramente a las especificaciones de la norma descrita. Sin embargo, la temperatura y humedad dentro de las placas petri permitieron un abundante desarrollo de micelio sobre las probetas testigo, por lo que se estima que éstas condiciones no afectaron significativamente este ensayo.

Las variaciones en el grado de ataque entre las probetas de una misma concentración se registró también en un ensayo paralelo según el método de los discos, en el que se utiliza madera seca inmpregnada en una solución de Agar al $1 \%$, lo que garantiza la homogeneidad del sustrato (Peredo, 1980). Esto se podría explicar por la relación, no siempre clara, entre concentración aplicada y preservante retenido, datos que constrastan con la baja desviación estándar calculada para la absorción y retención de preservantes evaluada en el estudio de Peña (1988).

Como las características del baño antimancha fueron las mismas para todas las probetas tratadas, se podría suponer que, como el tamaño y corte de las probetas permiten que éstas tengan la superficie total de una cara con madera temprana o tardia, la absorción y retención de las soluciones preservantes no fue homogénea. Esto se podria solucionar utilizando probetas de corte radial, que incluyen madera temparan y tardía en una misma cara, o elaborando probetas de corte transversal, de tablas o ramas, como las que utilizó originalmente Schulz (1951) en el método de los discos. 


\section{Epoca de ensayo}

La fecha en que se realizó la prueba de eficacia en terreno corresponde a la época de menos lluvia y mayor calor en la ciudad de Valdivia. Los cuatro dias siguientes a la instalación del ensayo fueron muy cálidos y con fuerte irradiación solar. Esto debió disminuir el contenido de humedad de la madera en forma considerable, desfavoreciendo el desarrollo de los hongos manchadores a través de todo el ensayo.

La época de ensayo puede introducir otros factores de variación, como la concentración de inóculo en el ambiente y su composición, y el contenido de nutrientes disponibles para los hongos manchadores en la maera. Keirle (1977) observó que la época de mayor susceptibilidad de las trozas de pino insigne al ataque de hongos manchadores y pudridores es en primavera y verano, y sugiere que esto se relaciona con la variación estacional del contenido de nitrógeno en la maera de albura.

\section{Diseño experimental}

El diseño del encastillado, donde las concentraciones menores fueron apiladas sobre las mayores, eliminó el riesgo de que una posible lixiviación de los productos aumentara la concentración del preservante en la maera ubicada abajo. Sin embargo, este diseño plantea dos interrogantes. :

Las concentraciones menores pudieron tener una mayor lixiviación por estar ubicadas en la parte superior del castillo, expuestas al efecto directo de la lluvia. Esto explicaría el quiebre de la curva que relaciona la superficie manchada con la concentración de SINESTO B, cuando este producto se aplica al $4,5 \%$ en manera apilada con separador (Figura $N^{\circ} 3$ ). Se pudo observar que las probetas tratadas a esta concentración fueron atacadas con mayor intensidad, en la medida en que se encontraban más cerca de la superficie superior del castillo. 
En el bloque superior de la madera sin tratamiento (Figura $\mathrm{N}^{\circ} 1$ ), el ataque de hongos manchadores en las probetas encastilladas con separador es considerablemente mayor, al compararlo con el bloque inferior. Esto se debe, seguramente, a la diferencia de temperatura, humedad y radiación solar que existe al acercarse o distanciarse del suelo.

Estos dos posibles sesgos en los resultados pudieron ser evitados, apilando la madera tratada con cada concentración en castillos independientes.

\section{Hongos manchadores}

La utilización de Ceratocystis pilifera como hongo de prueba en el ensayo de laboratorio, se basa en su descripción como principal agente causante de mancha azul en madera de pino en el país (Aguilar, 1985). Sin embargo se se sabe que cada año siguien llegando nuevos hongos, debido las importaciones de productos, turismo, etc. (Osorio, 1993)( ${ }^{(*)}$. Estos, y los demás hongos manchadores que se conoce en Chile, junto a innumerables microorganismos. crean un complejo sistema de interacción: entre ellos, con la madera tratada, y con el producto aplicado, llegando incluso a reducir el nivel de efectividad de un fungicida aplicado (Peredo, 1993) ${ }^{(*)}$, dada la capacidad de algunas bacterias de causar su propia biodetoxificación (Williams, 1991a).

De esta manera se podría explicar, que la influencia de la concentración del fungicida no fuera tan determinante en el azulado de la madera en condiciones naturales, como lo fue en la prueba de eficacia en laborarorio.

De esta manera se podria explicar, que la influencia de la concentración del fungicida no fuera tan determinante en el azulado de la madera en condiciones naturales, como lo fue en la prueba de eficacia en laboratorio.

Se puede suponer que, aparte de la influencia de la época de ensayo, el tiempo de almacenamiento de las trozas en el bosque y en el aserradero hacen variar el tipo y cantidad de agentes manchadores y organismos asociados, que influyen en la agresividad del ataque y sobre la eficacia del preservante, dado

(*) Dr. Moisés Osorio, U.A.Ch. Comunicación personal.

("*) Dr. Hernán Peredo, U.A.Ch. Comunicación personal. 
que los trozos traídos del bosque contienen altos niveles de desarrollo fungoso, que si no causan daño directo al rollizo, son una importnte fuente de inóculo (Williams, 1991b)

Todas estas consideraciones, hacen recomendable completar una comparación de eficacia entre pruebas in vitro $e$ in situ con ensayos realizados en otras épocas del año.

\section{Resultados obtenidos}

Como se ha señalado, la pendiente de la curva de superficie manchada es menos pronunciada en terreno que en laboratorio. Por ésto, la comparación de la dosis minima efectiva, tiene un factor de variación : la superficie manchada que se tolera. Si por ejemplo, este límite de tolerancia baja muy cerca de cero, la d.m.e. en terreno puede elevarse cerca de un punto en este ensayo.

Otro criterio que podría haber cambiado los resultados de la comparación in vitro e in situ es la duración de cada uno de los ensayos realizados, ya que el desarrollo de la mancha azul en el tiempo puede ser distinto en laboratorio que en terreno. Se podria esperar que la efectividad de los preservantes permanezca por menor tiempo en terreno, debido a los efectos del clima y los microorganismos. Pero, por otro lado, las óptimas condiciones de cultivo en laboratorio, donde el hongo crece además sin competencia, podrian permitir un ataque más intenso.

\section{CONCLUSIONES}

Los productos BUSAN 1009 y SINESTO B fueron efectivos al $2,0 \%$ y al $5,0 \%$ de concentración respectivamente, en la prevención del ataque de Ceratocystis pilifera (Fries) C. Moreau sobre madera de Pinus radiata D. Don, según las especificaciones de la norma ASTM D 4445-84.

En la prueba de eficacia realizada en terreno, la d.m.e. de estos productos nunca fue mayor que en el ensayo montado en laboratorio. En la madera 
tratada con BUSAN 1009, encastillada sin separador y con SINESTO B, encastillada con separador, la d.m.e. resultó ser la misma. En los demás casos, la fue menor.

La eficacia de los productos antimancha utilizados en terreno fue mayor en las concentraciones bajas y menor en las concentraciones altas, al compararla con los resultados obtenidos en laboratorio. Sin embargo, dentro del contexto explicado al comienzo de este trabajo, se puede concluir a partir de su desarrollo experimental, que existe una clara asociación entre la eficacia de los dos productos analizados en laboratorio y en terreno.

La madera encastillada sin separador fue atacada con mucha mayor intensidad que la madera apilada con separadores, especialmente en las probetas testigo.

\section{REFERENCIAS BIBLIOGRAFICAS}

ASTM., 1984. Standard method for testing fungicides for controlling sapstain and mold on unseasoned lumber (laboratory method). Annual Book of ASTM Standards. Vol 1101: 705-710

C.E.E., 1991. Norma del Consejo del 21 de Marzo de $1991.9^{\circ}$ modificación de la norma 76/769/CEE. (91/173/CEE).

David, R., 1991. La posición del pentaclorofenato de sodio en el contexto europeo. In Conceptualización de los eventuales productos antimancha en madera aserrada. Hickson Quimetal Latino América Ltda. Santiago, Chile. Marzo de 1991.

Hanke, T., 1991. Cinco años de investigación en Chile en la identificación de productos alternativos al pentaclorofenato de sodio en tratamientos de madera. In Conceptualización de los eventuales productos antimancha en madera aserrada. Hickson Quimetal Latino América Ltda. Santiago, Chile. Marzo de 1991.

Keirle, R., 1977. Effect of storage in different seasons on sapstain and decay of Pinus radiata D. Don. in N.S.W. Aust. For., 1978. 41 (1) : 29-36. 
Peña, R. 1988. Determinación de la eficacia de productos antimancha sin fenoles clorados en su formulación. Tesis Ing. Forestal. Universidad Austral de Chile, Valdivia, $35 \mathrm{p}$.

Peredo, M., 1980. Determinación de la eficacia de algunos preservantes antimancha Publicación Técnica $N^{\circ} 5$. Universidad Austral de Chile. Facultad de Ciencias Forestales $14 \mathrm{p}$.

Quiroz, I., 1991. Control biológico in vitro de hongos biodeterioradores de la madera de Pinus radiata $D$. Don presentes en Chile. Tesis Ing. Forestal. Universidad Austral de Chile. 29 p.

Schulz, G., 1951. Ein mykologisches Verfahren zur Bewertung vorbeugender Schutzmittel gegen das Verblauen von Kiefernholz. Angew. Bot 26 : 42-54.

Williams, G., 1991a. La biodegradación de la madera aserrada por la acción de microorganismos. In Conceptualización de los eventuales productos antimancha en madera aserrada. Hickson Quimetal Latino América Ltda. Santiago, Chile. Marzo de 1991.

Williams, G., 1991b. El desarrollo de compuestos antimancha alternativos y factores que afectan su grado de efectividad In : Conceptualización de los eventuales productos antimancha en madera aserrada. Hickson Quimetal Latino América Ltda. Santiago, Chile. Marzo de 1991 